\title{
Phenomenological Implications of a Class of Lepton Mass Matrices
}

\author{
S. Dev ${ }^{*, 1}$, Sanjeev Kumar ${ }^{\dagger, 2}$, Surender Verma ${ }^{\ddagger}, 1$ and Shivani Gupta ${ }^{\S, 1}$ \\ ${ }^{1}$ Department of Physics, Himachal Pradesh University, Shimla \\ 171005, INDIA. \\ ${ }^{2}$ School of Physics and Material Science, Thapar University, Patiala \\ 147004 , INDIA.
}

\begin{abstract}
Phenomenological implications of a class of lepton mass matrices with parallel texture structure have been examined and phenomenologically interesting constraints on charged lepton and neutrino mass matrix parameters have been obtained.
\end{abstract}

\section{INTRODUCTION}

Mass matrices provide important tools for the investigation of the underlying symmetries and the resulting dynamics. The first step in this direction is the reconstruction of the neutrino mass matrix in the flavor basis. However, the reconstruction results in a large variety of possible structures of mass matrices depending strongly on the mass scale, mass hierarchy and the Majorana phases. In the flavor basis, the mass matrix for Majorana neutrinos contains nine physical parameters viz. three mass eigenvalues, three mixing angles and the three $\mathrm{CP}$-violating phases. The two squared-mass differences $\left(\Delta m_{12}^{2}\right.$ and $\left.\Delta m_{13}^{2}\right)$ and the two mixing angles $\left(\theta_{12}\right.$ and $\left.\theta_{23}\right)$ have been measured in solar, atmospheric and reactor experiments. The third mixing angle $\theta_{13}$ and the Dirac-type CP-violating phase $\delta$ are expected to be measured in the forthcoming neutrino oscillation experiments. The possible measurement of the effective Majorana mass in neutrinoless double decay searches will provide an additional constraint on the remaining three neutrino parameters viz. the neutrino mass scale and two

*dev5703@yahoo.com

†sanjeev.verma@thapar.edu

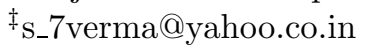

§shiroberts_1980@yahoo.co.in 
Majorana CP-violating phases. While the neutrino mass scale will be independently determined by the direct beta decay searches and cosmological observations, the two Majorana phases will not be uniquely determined even if the absolute neutrino mass scale is known. Thus, it is not possible to fully reconstruct the neutrino mass matrix with the observations from the presently feasible experiments. Under the circumstances, it is natural to employ other theoretical inputs for the reconstruction of the neutrino mass matrix. The possible forms of these additional theoretical inputs are constrained by the existing neutrino data. Several proposals have been made in the literature to restrict the form of the neutrino mass matrix by reducing the number of free parameters which include presence of texture zeros [1, 2, ㄱ, 4, [5, 6, 7, 8], requirement of zero determinant [9, 10] and the zero trace condition [11] amongst others. Some attempts aimed at understanding the pattern of quark/lepton masses and mixings by introducing Abelian or non-Abelian flavor symmetries, naturally, lead to texture zeros in the mass matrices. However, the current low energy data are consistent with a limited number of texture zero schemes [1, 2, 3, 4, 5, 6, 7, 8]. In particular, the latest low energy data disfavor [12] all quark mass matrices with five or more texture zeros. Four texture zero Ansätze is specially important since it can successfully describe not only the quark but also the lepton sector including the charged lepton and neutrino masses. Furthermore, not all of the texture zeros in the four texture zero Ansätze can be obtained through weak basis transformations and, therefore, have physical implications. In the present work, we examine a special case of four texture zero Ansätze in which the charged lepton mass matrix and Majorana neutrino mass matrix have the same texture zero structure with zero entries at $(1,1)$ and $(1,3)$ places. This Ansätze has been analyzed earlier [13] under the assumption of factorizable phases in the neutrino mass matrix which, however, is not always possible for a general complex symmetric matrix without unnatural fine tuning of the phases. In the context of the Type II see-saw mechanism for example, the phases of the Dirac neutrino mass matrix and the right handed Majorana neutrino mass matrix are assumed to be fine tuned to achieve the cancellation of phases in most of the analyses [14, 15] (and references therein) reported hitherto. In this sense, the analyses reported so far are incomplete. The condition of factorizable phases in the neutrino mass matrix was relaxed in [16]. However, this analysis was confined to the investigation of neutrino hierarchy and effective Majorana mass. In the present work, we have attempted an exhaustive analysis of a class of four-zero texture lepton mass matrices by examining its full implications for the lepton mass matrices.

All the information about lepton masses and mixings is encoded in the hermitian charged lepton mass matrix $M_{l}$ and the complex symmetric neutrino mass matrix $M_{\nu}$. In the present work, we consider a special case of four texture zero Ansätze in which the charged lepton mass matrix and Majorana neutrino mass matrix have the same texture zero structure with zero entries at $(1,1)$ and $(1,3)$ places and are given by

$$
M_{l}=\left(\begin{array}{ccc}
0 & A_{l} & 0 \\
A_{l} & D_{l} & B_{l} \\
0 & B_{l} & C_{l}
\end{array}\right)
$$


and

$$
M_{\nu}=\left(\begin{array}{ccc}
0 & A_{\nu} & 0 \\
A_{\nu} & D_{\nu} & B_{\nu} \\
0 & B_{\nu} & C_{\nu}
\end{array}\right)
$$

respectively. The hermiticity of $M_{l}$ requires its diagonal elements $D_{l}$ and $C_{l}$ to be real whereas the non-diagonal elements $A_{l}, B_{l}$ are, in general, complex with $A_{l}=\left|A_{l}\right| e^{i \phi_{1}}$, $B_{l}=\left|B_{l}\right| e^{i \phi_{2}}$. In contrast, all the non-vanishing elements of the complex symmetric matrix $M_{\nu}$ are, in general, complex.

The hermitian mass matrix, $M_{l}$ for the charged lepton, is diagonalized by the unitary transformation

$$
M_{l}=V_{l} M_{l}^{d} V_{l}^{\dagger}
$$

where $V_{l}^{\dagger}=V_{l}^{-1}$. The hermitian matrix $M_{l}$ can, in general, be written as

$$
M_{l}=P_{l} M_{l}^{r} P_{l}^{\dagger}
$$

where $P_{l}$ is a unitary diagonal phase matrix, $\operatorname{diag}\left(e^{i \phi_{1}}, 1, e^{i \phi_{2}}\right)$, and $M_{l}^{r}$ is real matrix which can be diagonalized by a real orthogonal matrix $O_{l}$ :

$$
M_{l}^{r}=O_{l} M_{l}^{d} O_{l}^{T}
$$

where the superscript $\mathrm{T}$ denotes transposition and $M_{l}^{d}=\operatorname{diag}\left(m_{e},-m_{\mu}, m_{\tau}\right)$. From Eqs.(3-5), the unitary matrix $V_{l}$ is given by

$$
V_{l}=P_{l} O_{l}
$$

Using the invariants, $\operatorname{Tr} M_{l}^{r}, \operatorname{Tr} M_{l}^{r^{2}}$ and $\operatorname{Det} M_{l}^{r}$, the matrix elements $\left|A_{l}\right|,\left|B_{l}\right|$ and $C_{l}$ can be written in terms of the charged lepton masses and $D_{l}$ as

$$
\begin{array}{r}
C_{l}=m_{e}-m_{\mu}+m_{\tau}-D_{l}, \\
\left|A_{l}\right|=\left(\frac{m_{e} m_{\mu} m_{\tau}}{C_{l}}\right)^{\frac{1}{2}}, \\
\left|B_{l}\right|=\left[\frac{\left(m_{\tau}-m_{\mu}-D_{l}\right)\left(m_{\tau}+m_{e}-D_{l}\right)\left(m_{\mu}-m_{e}+D_{l}\right)}{C_{l}}\right]^{\frac{1}{2}} .
\end{array}
$$

Here, $D_{l}$ should be in the range $\left(m_{e}-m_{\mu}\right)<D_{l}<\left(m_{\tau}-m_{\mu}\right)$ for the elements $\left|A_{l}\right|$ and $\left|B_{l}\right|$ to be real.

Using Eq. (7), the elements of the diagonalizing matrix, $O_{l}$ can be written in terms of charged lepton masses $m_{e}, m_{\mu}, m_{\tau}$ and charged lepton mass matrix element $D_{l}$, 
which are given by

$$
\left.\begin{array}{c}
O_{11}=\sqrt{\frac{m_{\mu} m_{\tau}\left(m_{\tau}-m_{\mu}-D_{l}\right)}{\left(m_{e}-m_{\mu}+m_{\tau}-D_{l}\right)\left(m_{e}+m_{\mu}\right)\left(m_{\tau}-m_{e}\right)}} \\
O_{12}=\sqrt{\frac{m_{\tau} m_{e}\left(m_{\tau}+m_{e}-D_{l}\right)}{\left(m_{e}-m_{\mu}+m_{\tau}-D_{l}\right)\left(m_{e}+m_{\mu}\right)\left(m_{\tau}+m_{\mu}\right)}} \\
O_{13}=\sqrt{\frac{m_{e} m_{\mu}\left(m_{\mu}-m_{e}+D_{l}\right)}{\left(m_{e}-m_{\mu}+m_{\tau}-D_{l}\right)\left(m_{\mu}+m_{\tau}\right)\left(m_{\tau}-m_{e}\right)}} \\
O_{21}=\sqrt{\frac{m_{e}\left(m_{\tau}-m_{\mu}-D_{l}\right)}{\left(m_{e}+m_{\mu}\right)\left(m_{\tau}-m_{e}\right)}} \\
O_{22}=\sqrt{\frac{m_{\mu}\left(m_{\tau}+m_{e}-D_{l}\right)}{\left(m_{\mu}+m_{\tau}\right)\left(m_{e}+m_{\mu}\right)}} \\
O_{31}=\sqrt{\frac{m_{\tau}\left(m_{\mu}-m_{e}+D_{l}\right)}{\left(m_{\tau}-m_{e}\right)\left(m_{\mu}+m_{\tau}\right)}} \\
O_{32}=\sqrt{\frac{m_{e}\left(m_{\mu}-m_{e}+D_{l}\right)\left(m_{\tau}+m_{e}-D_{l}\right)}{\left(m_{e}-m_{\mu}+m_{\tau}-D_{l}\right)\left(m_{\mu}+m_{e}\right)\left(m_{\tau}-m_{e}\right)}} \\
O_{33}=\sqrt{\frac{m_{\tau}\left(m_{l}\right)\left(m_{\tau}-m_{\mu}-m_{\mu}-D_{l}\right)}{\left(m_{e}-m_{\tau}\right)\left(m_{\tau}+m_{e}-m_{l}\right)}}
\end{array}\right\}
$$

If $D_{l}$ is known the diagonalizing matrix $O_{l}$ and the charged lepton mass matrix $M_{l}^{r}$ are fully determined since the charged lepton masses are known.

The complex symmetric neutrino mass matrix, $M_{\nu}$ is diagonalized by an orthogonal matrix, $V_{\nu}$

$$
M_{\nu}=V_{\nu} M_{\nu}^{\operatorname{diag}} V_{\nu}^{T}
$$

The neutrino mixing matrix or Pontecorvo-Maki-Nakagawa-Sakata matrix, $U_{P M N S}$, is given by

$$
U_{P M N S}=V_{l}^{\dagger} V_{\nu}
$$

The neutrino mixing matrix $U_{P M N S}$ consists of three non trivial CP-violating phases: the Dirac phase, $\delta$, and two Majorana phases, $\alpha$ and $\beta$, and, three neutrino mixing angles viz. $\theta_{12}, \theta_{23}$ and $\theta_{13}$. The neutrino mixing matrix can be written as product of two matrices characterizing Dirac and Majorana type $\mathrm{CP}$ violation

$$
U_{P M N S}=U P
$$

where $U$ and $P$ are given by

$U=\left(\begin{array}{ccc}c_{12} c_{13} & s_{12} c_{13} & s_{13} e^{-i \delta} \\ -s_{12} c_{23}-c_{12} s_{23} s_{13} e^{i \delta} & c_{12} c_{23}-s_{12} s_{23} s_{13} e^{i \delta} & s_{23} c_{13} \\ s_{12} s_{23}-c_{12} c_{23} s_{13} e^{i \delta} & -c_{12} s_{23}-s_{12} c_{23} s_{13} e^{i \delta} & c_{23} c_{13}\end{array}\right), P=\left(\begin{array}{ccc}1 & 0 & 0 \\ 0 & e^{i \alpha} & 0 \\ 0 & 0 & e^{i(\beta+\delta)}\end{array}\right)$.

Using Eqs. (9) and (10), the neutrino mass matrix $M_{\nu}$ can be written as

$$
M_{\nu}=P_{l} O_{l} U_{P M N S} M_{\nu}^{\text {diag }} U_{P M N S}^{T} O_{l}^{T} P_{l} .
$$

The two zero textures in $M_{\nu}$ yield two complex equations viz.

$$
m_{1} a^{2}+m_{2} b^{2} e^{2 i \alpha}+m_{3} c^{2} e^{2 i(\beta+\delta)}=0
$$


and

$$
m_{1} a d+m_{2} b g e^{2 i \alpha}+m_{3} c h e^{2 i(\beta+\delta)}=0
$$

where the complex coefficients $a, b, c, d, g$ and $h$ are given by

$$
\begin{aligned}
a & =O_{11} U_{e 1}+O_{12} U_{m 1}+O_{13} U_{t 1}, \\
b & =O_{11} U_{e 2}+O_{12} U_{m 2}+O_{13} U_{t 2}, \\
c & =O_{11} U_{e 3}+O_{12} U_{m 3}+O_{13} U_{t 3}, \\
d & =O_{31} U_{e 1}+O_{32} U_{m 1}+O_{33} U_{t 1}, \\
g & =O_{31} U_{e 2}+O_{32} U_{m 2}+O_{33} U_{t 2}, \\
h & =O_{31} U_{e 3}+O_{32} U_{m 3}+O_{33} U_{t 3} .
\end{aligned}
$$

Solving Eqs. (14-15) for the two mass ratios $\left(\frac{m_{1}}{m_{2}}\right)$ and $\left(\frac{m_{1}}{m_{3}}\right)$, we obtain

$$
\begin{gathered}
\frac{m_{1}}{m_{2}} e^{-2 i \alpha}=\frac{b(c g-b h)}{a(a h-c d)}, \\
\frac{m_{1}}{m_{3}} e^{-2 i \beta}=\frac{c(b h-c g)}{a(a g-b d)} e^{2 i \delta} .
\end{gathered}
$$

One can enumerate the number of parameters in Eqs. (17). The nine parameters (three neutrino mixing angles $\left(\theta_{12}, \theta_{23}, \theta_{13}\right)$, three neutrino mass eigenvalues $\left(m_{1}, m_{2}, m_{3}\right)$, two Majorana phases $(\alpha, \beta)$ and one Dirac-type CP violating phase, $\delta)$ come from neutrino sector and four parameters (three charged lepton masses $\left(m_{e}, m_{\mu}, m_{\tau}\right)$ and $\left.D_{l}\right)$ come from charged lepton sector, thus, totalling 13 parameters. The three charged lepton masses are [17]

$$
m_{e}=0.510998910 \mathrm{MeV}, m_{\mu}=105.658367 \mathrm{MeV}, m_{\tau}=1776.84 \mathrm{MeV} .
$$

The experimental constraints on the neutrino parameters at one standard deviation are [18]

$$
\begin{gathered}
\Delta m_{12}^{2}=7.67_{-0.21}^{+0.22} \times 10^{-5} \mathrm{eV}^{2} \\
\Delta m_{13}^{2}=(2.37 \pm 0.15) \times 10^{-3} \mathrm{eV}^{2} \\
\theta_{12}=34.5 \pm 1.4 \\
\theta_{23}=42.3_{-3.3}^{+5.1}
\end{gathered}
$$

Only an upper bound is known on the mixing angle $\theta_{13}$ from the CHOOZ experiment. However, the latest global analysis [19] gives

$$
\sin ^{2} \theta_{13}=0.016_{-0.01}^{+0.01}
$$

which is non-zero at $90 \%$ C.L.. The parameters $\delta$ and $D_{l}$ are varied uniformly within their full ranges. Thus, we are left with three unknown parameters viz. $m_{1}, \alpha, \beta$.

From Eqs. (17), two mass ratios $\left(\frac{m_{1}}{m_{2}}\right)$ and $\left(\frac{m_{1}}{m_{3}}\right)$ can be written as

$$
\frac{m_{1}}{m_{2}}=\left|\frac{b(c g-b h)}{a(a h-c d)}\right|,
$$




$$
\frac{m_{1}}{m_{3}}=\left|\frac{c(b h-c g)}{a(a g-b d)} e^{2 i \delta}\right| .
$$

For the simultaneous existence of two texture zeros at $(1,1)$ and $(1,3)$ positions in $M_{\nu}$, the two values of $m_{1}$ given by

$$
m_{1}=\left(\frac{m_{1}}{m_{2}}\right) \sqrt{\frac{\Delta m_{12}^{2}}{1-\left(\frac{m_{1}}{m_{2}}\right)^{2}}}
$$

and

$$
m_{1}=\left(\frac{m_{1}}{m_{3}}\right) \sqrt{\frac{\Delta m_{12}^{2}+\Delta m_{23}^{2}}{1-\left(\frac{m_{1}}{m_{3}}\right)^{2}}},
$$

calculated from the mass ratios $\left(\frac{m_{1}}{m_{2}}, \frac{m_{1}}{m_{3}}\right)$, respectively, must be identical. This constraint can be used to constrain the unknown variables $\delta, D_{l}$ and the third mixing angle $\theta_{13}$ which has not been measured experimentally as yet. We have, also, calculated the effective Majorana mass $M_{e e}$ appearing in the neutrinoless double beta decay for the allowed parameter space

$$
M_{e e}=m_{1}\left|U_{e 1}\right|^{2}+m_{2}\left|U_{e 2}\right|^{2}+m_{3}\left|U_{e 3}\right|^{2} .
$$

The Majorana phases $\alpha$ and $\beta$ are given by

$$
\begin{gathered}
\alpha=-\frac{1}{2} \arg \left(\frac{b(c g-b h)}{a(a h-c d)}\right), \\
\beta=-\frac{1}{2} \arg \left(\frac{c(b h-c g)}{a(a g-b d)} \times e^{2 i \delta}\right) .
\end{gathered}
$$

Using these relations, the Majorana phases $\alpha$ and $\beta$ have been calculated for the allowed values of $\delta, \theta_{13}$ and $D_{l}$.

\section{Results and Discussion}

The $\delta, \theta_{13}$ and $D_{l}$ parameter space allowed by the current neutrino oscillation data have been depicted in Fig. 1(a) and 1(b) as two dimensional scatter plots. We see that there are two distinct solutions in the $\left(\delta, \theta_{13}, D_{l}\right)$ parameter space. The more probable solution corresponds to lower values of $D_{l}$ and will be called "low $D_{l}$ " solution, henceforth. The less probable solution corresponds to higher values of $D_{l}$ and will be referred to as the "high $D_{l}$ " solution in the following discussion. In Fig. 1(c) and $1(\mathrm{~d}) M_{e e}-D_{l}$ and $\alpha-\beta$ correlation plots have been depicted. It is evident from Fig. 1 that these two solutions of $D_{l}$ have distinguishing implications, especially, for $\theta_{13}$. One of the characterizing feature of low $D_{l}$ solution is the existence of lower bound on $\theta_{13}$ i.e. $\theta_{13}>2.8^{\circ}$. The $95 \%$ C.L. ranges of $\theta_{13}$ and $D_{l}$ for these two solutions have been given in Table 1. Majorana mass term $M_{e e}$ is sharply constrained having 
values of the order of $10^{-3}$ for low $D_{l}$ solution, However, for high $D_{l}$ a wide range of $M_{e e}$ is allowed. The $95 \%$ C.L. ranges of $M_{e e}$ for these two solutions are tabulated in Table 1. Also, it can be inferred from Fig. 1 that Dirac-type CP-violating phase $\delta$ (Fig. 1(b)) and Majorana phase $\beta$ (Fig. 1(d)) remain unconstrained for low as well as high $D_{l}$ solution. However, Majorana phase $\alpha$ is constrained to the range $60^{\circ}-120^{\circ}$ for high $D_{l}$. In Fig. 2. we have plotted lightest neutrino mass eigenvalue $m_{1}$ with $\theta_{13}$ for low (left) and high $D_{l}$ (right) region. This plot has been reported earlier [16], however, no constraints have been obtained on $D_{l}$ and $\theta_{13}$. It is noted that the analysis done in [16] corresponds to low $D_{l}$ solution obtained in the present work. We have obtained an upper bound on $m_{1}$ of the order of $10^{-2}$ for low as well as high $D_{l}$ solution which is in agreement with that obtained in [16]. Also, we find that $m_{1}$ is bounded from below for low and high $D_{l}$ regions for $\theta_{13}<8^{\circ}$ and $<10^{\circ}$, respectively.

It should be noted that in performing numerical analysis we have used value of $\theta_{13}$ obtained from global analysis of neutrino data, given by Eq. (20). However, measurement of $\theta_{13}$ is the main goal of the future experiments on neutrino oscillations. The upcoming and proposed experiments in this context include Double CHOOZ [20, 21], Daya Bay [22, 23] and RENO [24]. Double CHOOZ is planned to explore $\sin ^{2} 2 \theta_{13}$ down to 0.06 in phase-I (0.03 in the later stages) and will be the first to report any observation on $\theta_{13}$. The Daya Bay experiment has a higher sensitivity and plans to observe $\sin ^{2} 2 \theta_{13}$ down to 0.01 . In this light it will be useful to relax the constraint on $\theta_{13}$ [Eq. (20)] and examine the phenomenological implications of this Ansätz for small $\theta_{13}$. We have considered three representative values of $\sin ^{2} 2 \theta_{13}$ viz. $0.01,0.03$ and 0.06 and examined the implications for the CP- violating phases $\alpha, \beta$ and $\delta$ depicted in Fig. 3 and Fig. 4, respectively, for low and high $D_{L}$ solutions. It can be seen from Fig. 3(a) and Fig. 3(b) that as $\sin ^{2} 2 \theta_{13}$ is decreased, the Majorana phases $\alpha, \beta$ becomes more constrained. Furthermore, Dirac-type CP-violating phase $\delta$ is constrained to narrower ranges as $\sin ^{2} 2 \theta_{13}$ is lowered which is evident from Fig. 4(a). However, for high $D_{l}$ solution $\delta$ remain unconstrained. There is no solution for low $D_{l}$ with $\sin ^{2} 2 \theta_{13}=0.01$ in Fig. 3 . and Fig. 4 because for low $D_{l}$ the third mixing angle $\theta_{13}>3^{\circ}$ which is, also, evident from Fig. 1(a).

\section{Conclusions}

In conclusion, we have considered a class of lepton mass matrices with parallel texture structure in charged lepton and Majorana neutrino mass matrices and obtained interesting constraints on the parameters of lepton mass matrices. An interesting feature of this analysis is the rather strong constraints obtained on $\theta_{13}$ and the emergence of two classes of solutions corresponding to 'low $D_{l}$ ' and 'high $D_{l}$ ' which is in contrast to the analysis reported earlier [16]. The implications of these two classes have been examined for the unknown parameters of the neutrino mass matrix like $\theta_{13}, M_{e e}$ and $\mathrm{CP}$-violating phases without the assumption of factorizable phases in the neutrino mass matrix. Some of the predictions of this analysis are testable in the forthcoming 
neutrino experiments.

\section{Acknowledgments}

The research work of S. D. is supported by the Board of Research in Nuclear Sciences (BRNS), Department of Atomic Energy, Government of India vide Grant No. 2004/ 37/ 23/ BRNS/ 399. S. K., S. G. and S. V. acknowledges the financial support provided by Council for Scientific and Industrial Research (CSIR) and University Grants Commission (UGC), Government of India, respectively.

\section{References}

[1] Paul H. Frampton, Sheldon L. Glashow and Danny Marfatia, Phys. Lett. B 536, 79 (2002).

[2] Bipin R. Desai, D. P. Roy and Alexander R. Vaucher, Mod. Phys. Lett A 18, 1355 (2003).

[3] Zhi-zhong Xing, Phys. Lett. B 530, 159 (2002).

[4] Wanlei Guo and Zhi-zhong Xing, Phys. Rev. D 67, 053002 (2003).

[5] Alexander Merle and Werner Rodejohann, Phys. Rev. D 73, 073012 (2006).

[6] S. Dev and Sanjeev Kumar, hep-ph/0607048.

[7] S. Dev, Sanjeev Kumar, Surender Verma and Shivani Gupta, Nucl. Phys. B 784, 103 (2007).

[8] S. Dev, Sanjeev Kumar, Surender Verma and Shivani Gupta, Phys. Rev. D 76, 013002 (2007).

[9] G. C. Branco, R. Gonzalez Felipe, F. R. Joaquim and T. Yanagida, Phys. Lett. B 562265 (2003).

[10] Bhag C. Chauhan, Joao Pulido and Marco Picariello, Phys. Rev. D 73, 053003 (2006).

[11] Xiao-Gang He and A. Zee, Phys. Rev. D 68, 037302 (2003), hep-ph/0302201 v2.

[12] Monika Randhawa, V. Bhatnagar, P. S. Gill, M. Gupta, Phys. Rev. D 60, 051301 (2005).

[13] Koichi Matsuda, Hiroyuki Nishiura, Phys. Rev. D 74, (2006) 033014.

[14] Gulsheen Ahuja, Sanjeev Kumar, Monika Randhawa, Manmohan Gupta, S. Dev, Phys. Rev. D 76, 013006 (2007). 
[15] Z. z. Xing and H. Zhang, Phys. Lett. B 569, 30 (2003).

[16] G. C. Branco, D. Emmanuel-Costa, R. González Felipe and H.Serôdio, JHEP 0801, 043 (2008), hep-ph/0711.1613v1.

[17] C. Amsler et al., Phys. Lett. B 667, 1 (2008).

[18] M. C. Gonzalez-Garcia, Michele Maltoni, Phys. Rept. 460 (2008) 1-129, hep$\mathrm{ph} / 0704.1800 \mathrm{v} 2$.

[19] G. L. Fogli, E. Lisi, A. Marrone, A. Palazzo, A. M. Rotunno, arXiv:hep$\mathrm{ph} / 0806.2649$.

[20] http://doublechooz.in2p3.fr/.

[21] F. Ardellier et al. (Double Chooz Collaboration), hep-ex/0606025.

[22] http://dayabay.ihep.ac.in/.

[23] Daya Bay Collaboration, hep-ex/0701029.

[24] http://neutrino.snu.ac.kr/RENO/. 


\begin{tabular}{|c|c|c|}
\hline Parameter & Low $D_{l}$ & High $D_{l}$ \\
\hline$\theta_{13}$ & $5.4^{\circ}-11.2^{\circ}$ & $3.4^{\circ}-11.2^{o}$ \\
$D_{l}$ & $(-85-415) \mathrm{MeV}$ & $(1555-1665) \mathrm{MeV}$ \\
$M_{e e}$ & $(1.0-1.5) \times 10^{-3} \mathrm{eV}$ & $(0.9-18.0) \times 10^{-4} \mathrm{eV}$ \\
\hline
\end{tabular}

Table 1: The ranges of $\theta_{13}, D_{l}$ and $M_{e e}$ at $95 \%$ C.L..
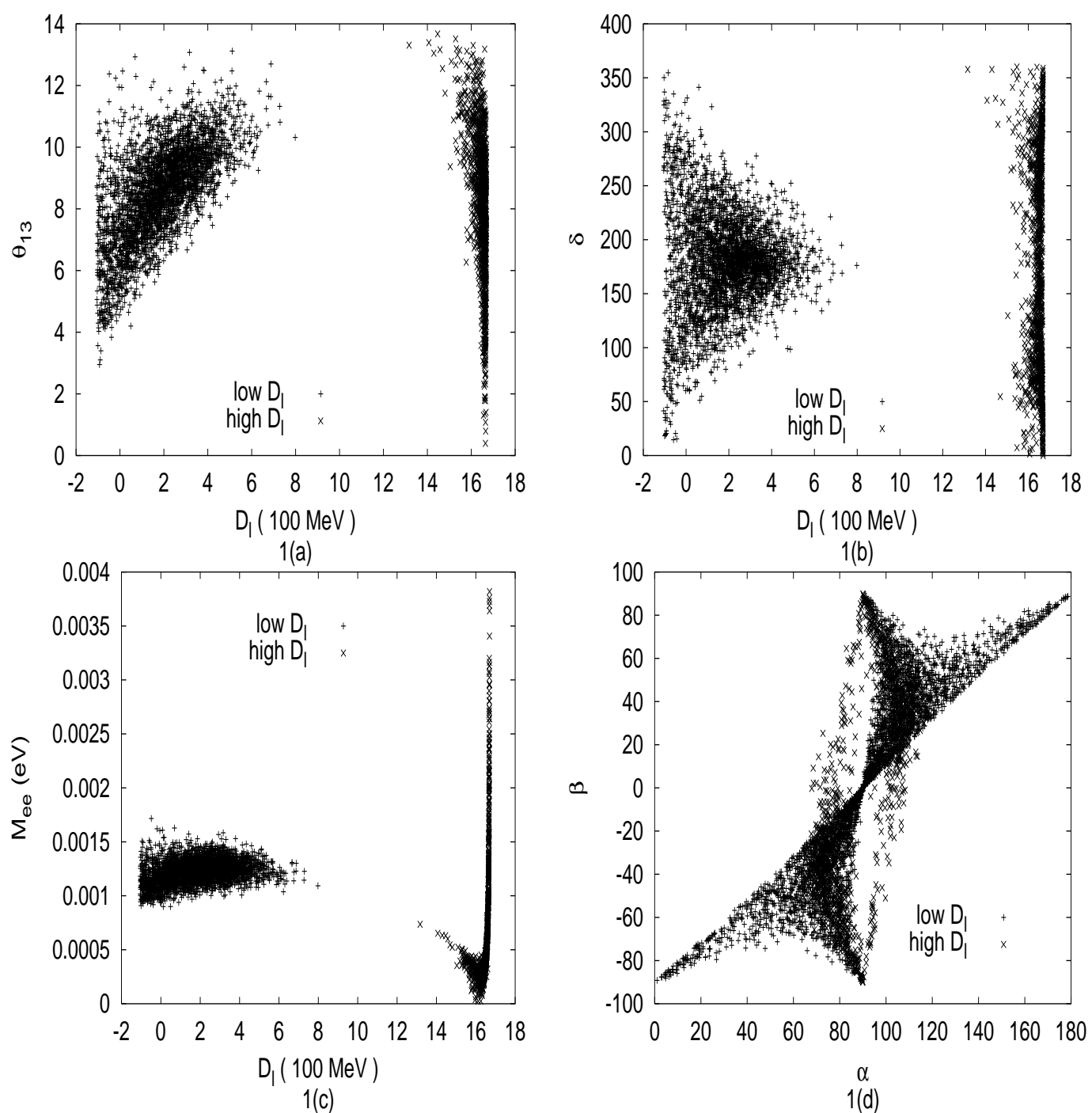

Figure 1: The parameter space allowed by the current neutrino oscillation data [18] and $\theta_{13}$ from global analysis [19]. The scatter plots of $\theta_{13}, \delta, M_{e e}$ with $D_{l}$ and of $\beta$ with $\alpha$. Low $D_{l}$ (high $D_{l}$ ) solution is represented by symbol 'plus' ('cross'). 

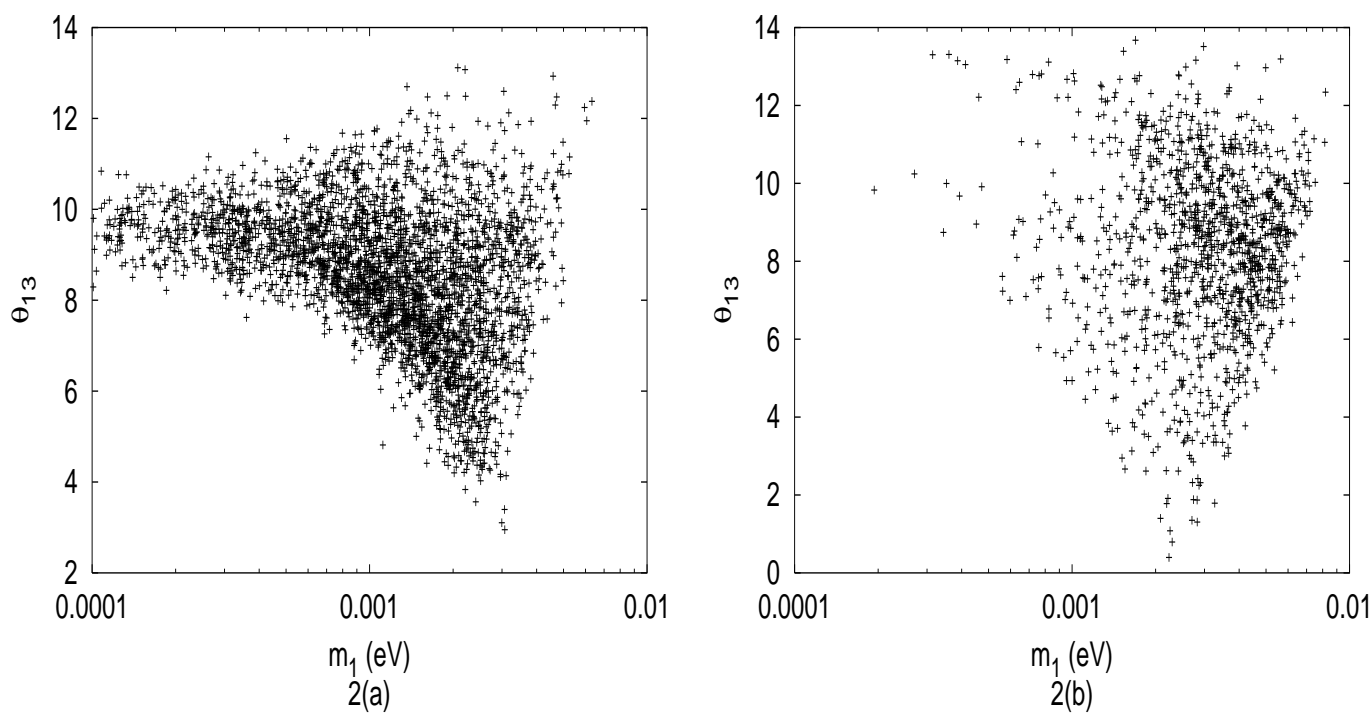

Figure 2: $\left(m_{1}-\theta_{13}\right)$ correlation plots for low $D_{l}$ (left) and high $D_{l}$ (right).
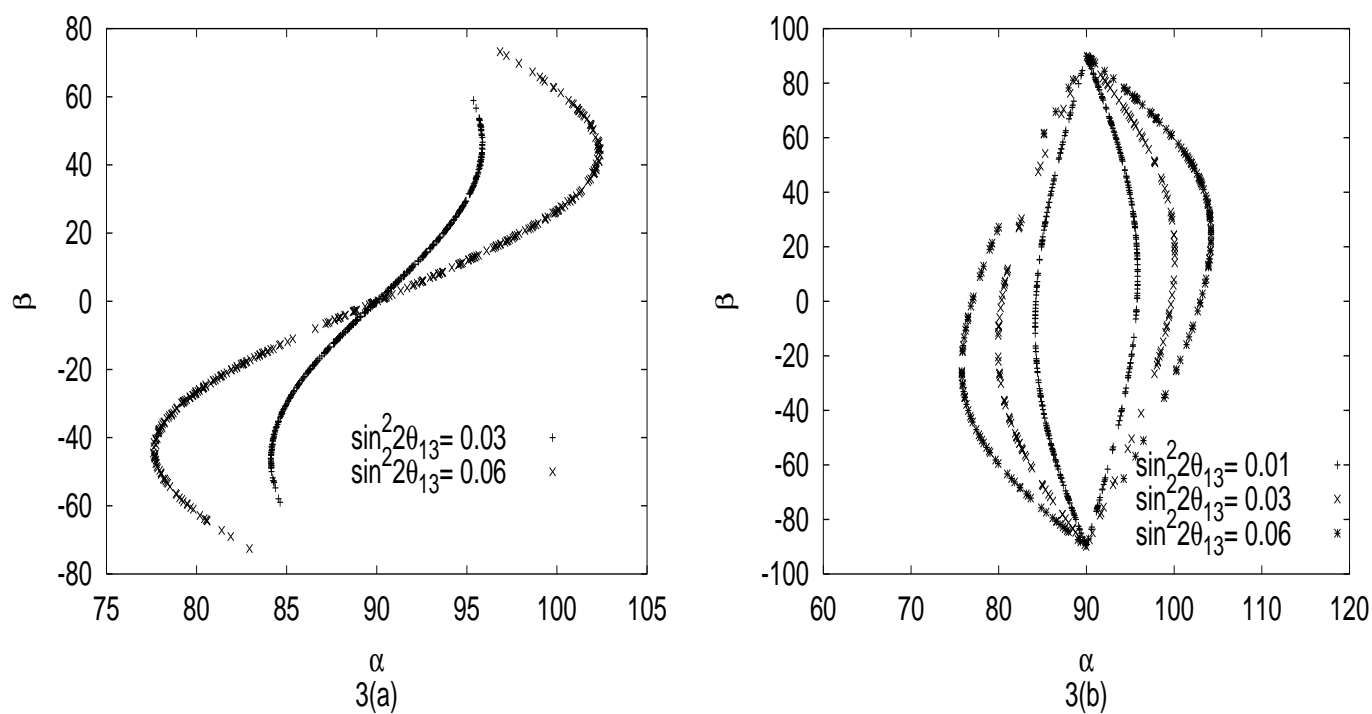

Figure 3: Correlation plot of the allowed points in $(\alpha-\beta)$ plane for low $D_{l}$ (left) and high $D_{l}$ region (right). 

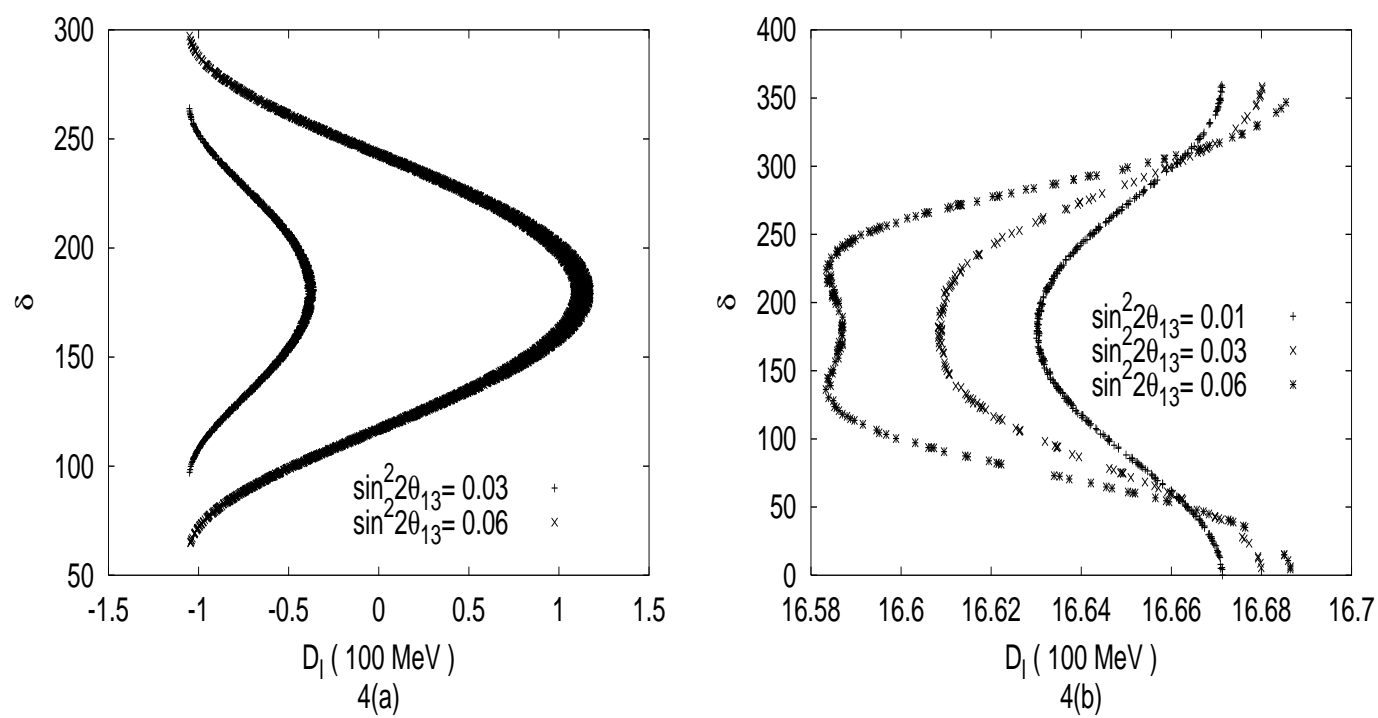

Figure 4: $\left(D_{l}-\delta\right)$ correlation plot for $\sin ^{2} 2 \theta_{13}=0.01, \sin ^{2} 2 \theta_{13}=0.03$ and $\sin ^{2} 2 \theta_{13}=$ 0.06 in low (left) and high (right) $D_{l}$ region. 